\title{
POINT SET OPERATORS AND THEIR INTERRELATIONS*
}

\author{
E. C. STOPHER, JR.
}

\section{INTRODUCTION}

The fundamental operators of this paper will be defined in terms of a postulated derived set function. This procedure, first formally suggested by F. Riesz, $\uparrow$ has been adopted by Chittenden $\ddagger$ and others.

The notation used here, which has definite advantages over the classical notation, was first suggested by Chittenden. It first appeared in print in work by Sanders§ and later in a paper by the author.\| Capital letters $A, B$, and so on, will denote arbitrary sets of points in the space $S, J(=c d S)$ will denote the isolated points of the space, and $0(=c S)$ the null set. Operators will be denoted by small letters $d, i$, and so on. Thus, for example, $i A$ represents the interior of the set $A$, and $d k A$ the derived set of the kernel of $A$.

Such an operator which defines a set, either null or non-null, will be called a product operator. The number of single operators which make up a product operator will be called the order of the product operator. A product operator is said to be reduced if it is shown to be equal to another product operator of lower order or to one of the same order but which is expressed in terms of operators which precede in the list of definitions in Part I. Those for which no reduction has been found will be called unreduced.

In Part I is presented a table of all the second order product operators indicating the reductions. Many other reduction formulas involving higher order operators have been found, but these will be omitted in this paper. II In Part II the space will be assumed to be dense in itself. With the aid of this additional postulate the theorem can be proved that all product operators of a given family can be expressed in a certain canonical form.

* Presented to the Society, April 9, 1937.

$\dagger$ F. Riesz, Stetigkeitsbegriff und abstrakte Mengenlehre, Atti del 4 Congresso Internationale dei Matematici, Rome, 1910, vol. 2, p. 18.

$\ddagger \mathrm{E}$. W. Chittenden, On general topology and the relation of the properties of the class of all continuous functions to the properties of space, Transactions of this Society, vol. 31 (1929), pp. 290-321.

$\S \mathrm{S}$. T. Sanders, Jr., Derived sets and their complements, this Bulletin, vol. 42 (1936), pp. 577-584.

\| E. C. Stopher, Jr., Cyclic relations in point set theory, this Bulletin, vol. 43 (1937), pp. 686-694.

I Found with proofs in the doctor's dissertation by the author, Interrelations of a Family of Operators on Point Sets and their Canonical Representation, State University of Iowa, 1937. 


\section{PART I. REDUCTION OF OPERATORS}

In the present discussion $d A$ is undefined and will be called the derived set of $A$. We postulate:

I. $d(A+B)=d A+d B$ (operator is additive).

II. $d^{2} A \leqq d A$ (derived sets are closed).

We make the following definitions:*

D 1. Complement. $c A=S-A$.

D 2. Extension. $e A=A+d A$.

D 3. Interior. $i A=A c d c A$.

D 4. Concentrated part. $h A=A d A$.

D 5. Isolated set. $j A=A c d A$.

D 6. Border. $b A=A d c A$.

D 7. Frontier. $f A=A d c A+c A d A$.

D 8. Kernel. $k A=\sum B \leqq A$, such that $B \leqq d B$.

D 9. Separated part. $s A=A c k A$.

The following table gives all second order operators $\phi_{1} \phi_{2} A$. The reductions are indicated and the unreduced operators are placed in parentheses.

TABLE I

\begin{tabular}{|c|c|c|c|c|c|c|c|c|c|c|}
\hline 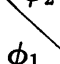 & $c$ & $d$ & $e$ & $i$ & $h$ & $j$ & $b$ & $f$ & $k$ & $s$ \\
\hline$c$ & 1 & $(c d)$ & $(c e)$ & $e c$ & $(c h)$ & $(c j)$ & $(c b)$ & $(c f)$ & $(c k)$ & $(c s)$ \\
\hline$d$ & $(d c)$ & $\left(d^{2}\right)$ & $d$ & $(d i)$ & $(d h)$ & $(d j)$ & $(d b)$ & $(d f)$ & $(d k)$ & $d j$ \\
\hline$e$ & $(e c)$ & $d$ & $e$ & $(e i)$ & $(e h)$ & $(e j)$ & $(e b)$ & $f$ & $d k$ & $e j$ \\
\hline$i$ & $c e$ & (id) & $(i e)$ & $i$ & $(i h)$ & $j i$ & 0 & (if) & $i h$ & $s i$ \\
\hline$h$ & $(h c)$ & $d^{2}$ & $d$ & (hi) & $\left(h^{2}\right)$ & 0 & $(h b)$ & $d f$ & $k$ & $s h$ \\
\hline$j$ & $(j c)$ & $(j d)$ & $j$ & $(j i)$ & $(j h)$ & $j$ & $(j b)$ & $(j f)$ & 0 & $j$ \\
\hline$b$ & $(b c)$ & $(b d)$ & (be) & 0 & $(b h)$ & $(b j)$ & $b$ & $(b f)$ & $(b k)$ & $(b s)$ \\
\hline$f$ & $f$ & $b d$ & $b e$ & $(f i)$ & $(f h)$ & $(f j)$ & $e b$ & $b f$ & $(f k)$ & $(f s)$ \\
\hline$k$ & $(k c)$ & $(k d)$ & $k d$ & $(k i)$ & $k$ & 0 & $(k b)$ & $(k f)$ & $k$ & 0 \\
\hline & $(s c)$ & $(s d)$ & $(s e)$ & $(s i)$ & $(s h)$ & $j$ & $(s b)$ & $(s f)$ & 0 & \\
\hline
\end{tabular}

One will note that of the 100 second order operators, 60 are unreduced. Of the 1000 third order operators 259 are unreduced. This decreasing rate of increase of the unreduced operators suggests that

* F. Hausdorff, Mengenlehre, Berlin, pp. 109-129. Under the postulates given, the extension function corresponds to Hausdorff's set of $\alpha$ points $A_{\alpha}$. The points of $d A$ correspond to his $\beta$ points $A_{\beta}$. Similarly, $h A$ corresponds to $A_{h}, j A$ to $A_{j}, b A$ to $A_{r}$ (border is a translation of the German word "rand"), $k A$ to $A_{k}$, and $s A$ to $A_{8 .}$. 
perhaps all product operators formed from these ten fundamental ones might be expressible in terms of some canonical system. This problem has not been solved for the general case.

It is interesting to note the simplifications which occur if we take the set $A$ to be the whole space $S$. The following table shows that there are only seven distinct first order operators $c S, d S, i S, j S, b S$, $k S$ and $s S$, and nine distinct unreduced second order ones. One might expect $b S(=S d c S)$ to be null, but it has not been assumed that the derived set of the null set is null.

TABLE II

\begin{tabular}{c|ccccccc}
$\phi_{1}{ }^{\boldsymbol{\phi}_{2} S}$ & $c S=0$ & $d S$ & $i S$ & $j S=J$ & $b S=d 0$ & $k S$ & $s S$ \\
\cline { 2 - 8 } & $S$ & $j S$ & $b S$ & $d S$ & $i S$ & $s S$ & $k S$ \\
$d$ & $b S$ & $\left(d^{2} S\right)$ & $d S$ & $(d j S)$ & $b S$ & $k S$ & $d j S$ \\
$e$ & $b S$ & $d S$ & $S$ & $(e j S)$ & $b S$ & $k S$ & $e j S$ \\
$i$ & $c S$ & $(i d S)$ & $i S$ & $j S$ & $c S$ & $i d S$ & $s S$ \\
$h$ & $c S$ & $d^{2} S$ & $(h i S)$ & $c S$ & $b S$ & $k S$ & $s d S$ \\
$j$ & $c S$ & $(j d S)$ & $j S$ & $j S$ & $c S$ & $c S$ & $j S$ \\
$b$ & $c S$ & $d j S$ & $c S$ & $c S$ & $b S$ & $(b k S)$ & $c S$ \\
$f$ & $b S$ & $d j S$ & $b S$ & $d j S$ & $b S$ & $b k S$ & $b k S$ \\
$k$ & $c S$ & $k S$ & $(k i S)$ & $c S$ & $b S$ & $k S$ & $c S$ \\
$s$ & $c S$ & $(s d S)$ & $s S$ & $j S$ & $c S$ & $c S$ & $s S$
\end{tabular}

Part II. SPaCe Dense In ITSElf

An additional postulate will be assumed in Part II:

III. $d S=S$.

Under Postulates I, II and III, a table similar to Table I would show 49 instead of 60 unreduced second order operators. There are 159 instead of 259 unreduced third order ones. A table similar to Table II under Postulates I, II, III would show only three distinct first order operators $c S, i S, b S$, and no unreduced second order operators.

Consider the family $\mathcal{E}$ consisting of the following sets, their complements, and these sets with $A$ replaced by $c A$ :

$$
\begin{array}{lr}
d^{\alpha} i d A, & \alpha=0 \text { or } 1, \\
h^{\alpha} c h^{\beta} A, & \alpha, \beta=1,2,3, \cdots \quad \text { (finite or transfinite ordinals), } \\
R(\alpha, \beta, \gamma, \kappa, \lambda, \mu, \nu)=k^{\alpha} s^{\beta} e^{\gamma} d^{\kappa} \prod_{\tau=1}^{\lambda}\left(j d^{a_{\tau}}\right) j^{\mu} h^{\nu} A,
\end{array}
$$


where, by definition, $\theta^{0} A=A, \prod_{\tau=1}^{0}\left(j d^{a_{\tau}}\right) A=A$, and $R$ is limited by the following equations:

$$
\begin{aligned}
\alpha, \beta, \gamma, \mu & =0 \text { or } 1, & \alpha \beta=0, & \alpha \gamma=0, \\
\alpha \kappa & =0 \text { or } 1, & \gamma \kappa=0, & \\
\lambda & =0,1,2, \cdots & & \text { (finite ordinals), } \\
\kappa, a_{\tau}, \nu & =0,1,2, \cdots & & \text { (finite or transfinite ordinals). }
\end{aligned}
$$

To illustrate, $R(1,0,0,1,0,1,5)=k d j h^{5} A$ and $R(0,0,1,0,2,0,1)$ $=e j d^{a_{1}} j d^{a_{2}} h A$.

We will designate by $\varepsilon_{1}$ the subset of $\varepsilon$ for which the parameters $\kappa$ and $a_{\tau}$ are restricted to be finite ordinals. The author has proved the following theorem:

TheOREM 1. The set $\phi A$, where $\phi$ is any finite product operator compounded from $c, d, e, i, h, j, k$ and $s$, is a set of $\varepsilon_{1}$.

The proof is one of mathematical induction. The first part of the proof consists of showing that the set $A$ is an $\varepsilon_{1}$ set. This is readily accomplished by noting that

$$
A=R(0,0,0,0,0,0,0),
$$

that is, $A$ is obtained as that special case of $R$ for which the value zero has been assigned to each of the parameters $\alpha, \beta$, and so on.

The second part of an ordinary proof by mathematical induction would consist of showing that the successor of a general set, $\phi A$, of the collection $\varepsilon_{1}$ is an $\varepsilon_{1}$ set. However, each of our sets has not just one but fourteen "successors" associated with it: $d \phi A, d c \phi A, e \phi A$, $e c \phi A, i \phi A, i c \phi A, h \phi A, h c \phi A, j \phi A, j c \phi A, k \phi A, k c \phi A, s \phi A$, and $s c \phi A$. One can omit $e c \phi A$ and $i c \phi A$ since $e c \phi A$ equals $c i \phi A$ and $i c \phi A$ equals $\operatorname{ce} \phi A$, but it is necessary to show that each of the other twelve "successors" of each of the $\varepsilon_{1}$ sets is an $\varepsilon_{1}$ set. This proof, which can be found in detail in the author's dissertation (loc. cit.), will be omitted here because of the large number of cases which must be considered.

The results have been extended to the following:

THEOREM 2. The set $\phi A$, where $\phi$ is any finite product operator compounded from $c, d, e, i, h, j, k$, s and their transfinite powers, is an $\mathcal{E}_{\text {set. }}$

Since $\theta^{2} A$ equals $\theta A$ or $A$ for all of the operators except $d$ and $h$, it is necessary to consider the transfinite powers of only these two. It is necessary to show that each $\varepsilon$ set when operated upon by transfinite powers of $d$ or of $h$ will yield an $\varepsilon$ set. For the details of this proof the reader is again referred to the same dissertation. 
It remains an unsolved problem whether or not generalizations of the theorems of this paper can be established which would include the border and frontier operators or which would not require that the space be dense in itself.

Ashland College

\section{A NOTE ON A PAPER BY J. A. TODD*}

\section{A. SINKOV}

In a recent paper $\dagger$ entitled $A$ note on the linear fractional group, Todd obtained an abstract definition for the group $L F\left(2,2^{n}\right)$ in terms of $n+2$ generators. Apparently he gave no consideration to the question of the independence of the defining relations, for they can be considerably simplified. First, in view of the condition $R S_{i}=S_{i+1} R$ (which is the same as $R^{i} S_{0} R^{-i}=S_{i}$ ), the three generators $U, R$ and $S_{0}$ are sufficient to generate the entire group. If we give a definition in ter $\mathrm{ms}$ of these three generators alone, the relations

$$
S_{i}^{2}=1, \quad i \neq 0, \quad R S_{i}=S_{i+1} R
$$

may be discarded, and any $S_{i}(i \neq 0)$ which appears in the remaining conditions may be replaced by its definition in terms of $R$ and $S_{0}$. Next, the $C_{n-1,2}$ conditions $S_{i} S_{j}=S_{j} S_{i}$ can be replaced by the $n-1$ conditions

$$
S_{0} S_{i}=S_{i} S_{0}, \quad i=1,2, \cdots, n-1 .
$$

For suppose $j-i=\alpha$. Then, from $S_{0} S_{\alpha}=S_{\alpha} S_{0}$, we get

$$
R^{i}\left(S_{0} S_{\alpha}\right) R^{-i}=R^{i}\left(S_{\alpha} S_{0}\right) R^{-i}, \quad S_{i} S_{j}=S_{j} S_{i} .
$$

Writing $S_{0} S_{i}=S_{i} S_{0}$ in terms of $R$ and $S_{0}$ only

$$
S_{0} R^{i} S_{0} R^{-i}=R^{i} S_{0} R^{-i} S_{0}, \quad\left(S_{0} R^{i} S_{0} R^{-i}\right)^{2}=1 .
$$

Thus, for the three generators $U, R$ and $S_{0}$, we require only $n+5$ conditions

$$
\begin{aligned}
R^{2^{n}-1}= & U^{3}=(U R)^{2}=\left(U S_{0}\right)^{2}=S_{0}{ }^{2}=1,\left(S_{0} R^{i} S_{0} R^{-i}\right)^{2}=1, \\
& R^{n} S_{0} R^{-n+1}=S_{0}^{a_{0}} R S_{0}^{a_{1}} R S_{0}^{a_{2}} \cdots R S_{0}^{a_{n-1}} R^{-n+2} .
\end{aligned}
$$

But even these three generators are not independent. For the relation $(U R)^{2}=1$ permits us to consider $U$ and $R$ as being equivalent to

* Presented to the Society, February 25, 1939.

$\dagger$ Journal of the London Mathematical Society, vol. 2 (1936), pp. 103-107. 